\title{
Dynamic Analysis of an Electoral Campaign $^{1}$
}

\author{
Luca Lambertini \\ Dipartimento di Scienze Economiche \\ Università di Bologna \\ Strada Maggiore 45, 40125 Bologna, Italy \\ fax +39-051-2092664 \\ phone $+39-051-2092600$ \\ e-mail lamberti@spbo.unibo.it
}

August 23, 2001

${ }^{1}$ I thank Roberto Cellini, Riccardo Rovelli and Sofia Ventura for helpful discussion. The usual disclaimer applies. 


\begin{abstract}
I analyse two differential games describing electoral campaigns where two candidates invest so as to increase the number of their respective voters. In both cases, parties overinvest and the number of voters is larger than in the social optimum. I extend both models to $n$ candidates, so as to derive the socially optimal number of candidates. This yields non-univocal results, in that the number of candidates maximising social welfare when a benevolent planner controls their efforts may be higher or lower than the optimal number of candidates given the non-cooperative investment behaviour of parties, according to the shape of cost functions and the dynamic behaviour of consensus associated with investments.
\end{abstract}

Keywords: elections, investment, consensus, differential games

JEL Classification: D72, M37 


\section{Introduction}

The aim of this paper consists in formalising electoral competition as a differential game where parties invest resources in the electoral campaign so as to increase the consensus for their respective candidates. Two parties noncooperatively invest or a finite horizon, with elections taking place at the terminal date, when the candidate receiveng the highest consensus wins the office. The campaigning activity is characterised by external effects, in the sense that part of each candidate's investment spills over to rivals. To model parties' investments during the campaign, two alternative technologies are considered. One involves a quadratic costs of investment at each point in time, while the amount of consensus evolves linearly over time. The other involves a linear cost of investment at each point in time, with decreasing returns to investment characterising the intertemporal evolution of consensus.

This setup allows me to address the following issues. First, whether parties invest too much in the electoral campaign as compared to what would be socially efficient. Relatedly, the second issue is whether maximising the number of voters (i.e., collective participation to the polls) is a sensible measure of welfare in such a setting, or not. This problem can be reformulated in equivalent but perhaps clearer terms by asking whether it is necessarily true that "the more people express their political opinions, the merrier we are". Indeed, the models presented in the remainder of the paper point to the opposite conclusion, as it appears that, since increasing the overall number of voters is costly, a social planner would maximise collective welfare by investing less in the electoral campaign, and having consequently a smaller number of voters at equilibrium, as compared to the private optimum generated by the non-cooperative behaviour of parties.

A third issue is whether it is possible to establish what the optimal number of parties should be, on welfare grounds. To address this topic, I generalise the model to account for $n$ parties, and assume for simplicity that all of them are a priori symmetric. Then, two alternative situations are envisaged. The first is social planning, where a benevolent dictator controls the advertising investment of each party, and maximises the collective steady state welfare level w.r.t. $n$. This is the first best solution. The second scenario is a private equilibrium driven by individual incentives, with the planner controlling only the number of parties, this being a second best solution. The answer of the model is not clear-cut. In general, the outcome is sentitive to the relative size of parameters as well as to the specific functional form assumed for the 
dynamics of accumulation of consensus. In particular:

- If the investment technology is characterised by quadratic instantaneous costs of investment, the socially optimal number of parties is higher (respectively, lower) than the number of parties at the second best solution, if the spillover effect is sufficiently large (low).

- If the investment technology is characterised by linear instantaneous costs of investment, the socially optimal number of parties is always lower than the number of parties at the second best, independently of the size of spillover effects.

The ensuing analysis is carried out by using dynamic models where the open-loop solution is subgame perfect. This is appealing since it means that agents rely upon simple decision rules adopted the very outset, and then strictly abided by along the game, as they are strictly time consistent. ${ }^{1}$

The remainder of the paper is organised as follows. Section 2 briefly discusses the related literature. The basic setup is laid out in section 3 . The alternative two-party models are investigated in sections 4-5. Sections 6-7 extend the analysis of both models to the case of $n$ parties. Section 8 contains concluding remarks.

\section{Relation to the literature}

Issues like the cost of campaigning, the search for funds, the value of incumbency and their relation with the outcome of elections have received a large amount of attention by economists and political scientists alike (Jacobsen, 1978, 1980, 1987; Baron, 1989; Abramovitz, 1991; Austen-Smith, 1995; Anderson and Prusa, 2001, to mention only a few). Indeed, Federal Election Commission data reveal the striking relevance of money in politics in general and electoral campaigns in particular. Candidates raising little or no money have negligible, if any, chances of winning.

To the best of my knowledge, this topics have never been explicitly put in relation with two connected streams of literature which are very familiar to

\footnotetext{
${ }^{1}$ The present paper shares this problem with the literature on economic policy. On this, as well as the growing attention to the interplay between economics and politics, see Persson and Tabellini (2000).
} 
industrial economists, namely those dealing with advertising and R\&D activities. Both kinds of investment relate to rent seeking behaviour, and they have been investigated extensively with and without uncertainty. ${ }^{2}$ Firms carry out advertising and $\mathrm{R} \& \mathrm{D}$ to acquire a dominat position on the market, either by increasing market share or by improving production technology. In doing so, some of each firm's effort spills over to rivals (see Brander and Spencer, 1983; Dixon, 1985). ${ }^{3}$ Profit incentives may give rise to overinvestment or underinvestment as compared to the social optimum, depending upon the shape of downstream market competition. ${ }^{4}$

Electoral campaigns share many fundamental features with advertising campaigns and R\&D races. Increasing consensus through costly investments is formally equivalent to acquiring a dominant market position through advertising or R\&D either in process or in product innovation. In doing so, each party may waste some amount of resources to the benefit of rivals, and the optimal investment effort as well as the outcome of elections will depend upon the size of such external effects. The only relevant difference is that the electoral campaign has a terminal date which is known a priori, while $\mathrm{R} \& \mathrm{D}$ races end at some uncertain date in the future, as soon as the first innovator obtains the new technology or product, and advertising campaigns may never end at all.

\section{Setup and definitions}

Consider a two-party system where each party has a candidate racing for the presidential office (or premiership), and elections are expected with certainty at date $T$. Over $t \in[0, T]$, each party invests in an advertising campaign so as to increase the number of votes to its candidate. Instantaneous investment is $k_{i}(t)$ while the number of votes, or the measure of consensus, is $x_{i}(t)$. In the jargon of optimal control theory, $k_{i}(t)$ is the control variable while $x_{i}(t)$

\footnotetext{
${ }^{2}$ Dynamic R\&D races under uncertainty are in Reinganum (1981, 1982a), Harris and Vickers $(1985,1987)$. A deterministic differential game of R\&D is in Cellini and Lambertini (2001). Comprehensive surveys on dynamic models of advertising can be found in Jørgensen (1982), Feichtinger, Hartl and Sethi (1994), Dockner, Jørgensen, Van Long and Sorger (2000).

${ }^{3}$ Advertising is often thought of as being a public good, in that firms may experience difficulties in internalising its beneficial effects (see Friedman, 1983; Fershtman, 1984; Fershtman and Nitzan, 1991).

${ }^{4}$ For exhaustive accounts, see Tirole (1988), Reinganum (1989) and Martin (1993).
} 
is the state variable. ${ }^{5}$

In the remainder of the paper, I will investigate two different dynamic equations for the state variable $x_{i}(t)$.

Model I Candidate $i^{\prime}$ s consensus evolves according to:

$$
\frac{\partial x_{i}(t)}{\partial t}=k_{i}(t)-s k_{j}(t)-\delta x_{i}
$$

where $s \in[0,1]$ is a negative spillover from candidate $j^{\prime}$ s investment onto candidate $i^{\prime}$ s consensus, and $\delta \in[0,1]$ is a constant depreciation rate. This is a slightly modified version of an advertising model described in Leitmann and Schmitendorf (1978) and Feichtinger (1983).

The gross instantaneous utility associated with consensus $x_{i}(t)$ is $\beta_{i} x_{i}(t)$, $\beta_{i}>0$, while the instantaneous cost of investment is

$$
C_{i}(t)=\frac{c}{2}\left[k_{i}(t)\right]^{2}
$$

Therefore, candidate $i$ aims at maximising

$$
\Pi_{i}=\int_{0}^{T} e^{-\rho t}\left\{\beta_{i} x_{i}(t)-\frac{c}{2}\left[k_{i}(t)\right]^{2}\right\} d t+e^{-\rho T} S\left[x_{i}(T)\right]
$$

under the constraint (1). The term $e^{-\rho T} S\left[x_{i}(T)\right]$ is the discounted scrap (or terminal) value of the state at the terminal date $T$.

Model II Candidate $i^{\prime}$ 's consensus evolves according to:

$$
\frac{\partial x_{i}(t)}{\partial t}=\sqrt{k_{i}(t)-s k_{j}(t)}-\delta x_{i}
$$

The gross instantaneous utility associated with consensus $x_{i}(t)$ is $\beta_{i} x_{i}(t)$, $\beta_{i}>0$. In this case, the instantaneous cost of investment is $C_{i}(t)=$ $c k_{i}(t)$. The discounted scrap value of the state at the terminal date $T$ is $e^{-\rho T} S\left[x_{i}(T)\right]$.

\footnotetext{
${ }^{5}$ In the present setting, the political platforms of parties is left unspecified. This issue is detailedly addressed in the literature using the spatial approach to multiparty competition. See Bartholdi, Narasimhan and Tovey (1991), Page, Kollman and Miller (1993), Anderson, Kats and Thisse (1994), Weber (1998), Adams (1999, 2000) Ansolabehere and Snyder (2000), inter alia.
} 
The main difference between model I on one side and model II on the other is in the way decreasing returns to advertising expenditure are introduced. While in model I decreasing returns to investment are modelled through a quadratic instantaneous cost, in model II they appear in the dynamic equation governing the evolution over time of the stock of votes accruing to candidate $i$.

\section{Model I}

When consensus accumulates according to (1), the relevant closed-loop Hamiltonian for party (or candidate) $i$ is:

$$
\begin{aligned}
\mathcal{H}_{i}(t)= & e^{-\rho t}\left\{\beta_{i} x_{i}(t)-\frac{c}{2}\left[k_{i}(t)\right]^{2}+\lambda_{i i}(t)\left[k_{i}(t)-s k_{j}(t)-\delta x_{i}\right]\right. \\
& \left.+\lambda_{i j}(t)\left[k_{j}(t)-s k_{i}(t)-\delta x_{j}\right]\right\}
\end{aligned}
$$

where $\lambda_{i i}(t)=\mu_{i i}(t) e^{\rho t}$, and $\mu_{i i}(t)$ is the co-state variable associated to $x_{i}(t)$. The supplementary variable $\lambda_{i i}(t)$ is introduced to ease calculations as well as the remainder of the exposition.

\subsection{The private optimum}

Here I investigate the outcome of the noncooperative game where each party maximises its own discounted (constrained) utility. On the basis of (5), the following holds:

Lemma 1 The open-loop equilibrium is a degenerate closed-loop equilibrium. Therefore, the open-loop equilibrium is subgame perfect.

Proof. See the Appendix.

Lemma 1 amounts to saying that this game is perfect or state redundant (see Mehlmann and Willing, 1983; Fershtman, 1987), ${ }^{6}$ and can be solved open-loop to obtain a strongly time-consistent equilibrium.

The open-loop solution leads to the following result:

\footnotetext{
${ }^{6}$ For the detailed analysis of several classes of games where open-loop equilibria are subgame perfect, see also Clemhout and Wan (1974), Reinganum (1982b), Dockner, Feichtinger and Jørgensen (1985), Fershtman, Kamien and Muller (1992). An exhastive exposition can be found in Başar, and Olsder (1982, 1995²), Mehlmann (1988, ch. 4) and Dockner, Jørgensen, Van Long and Sorger (2000, ch. 7).
} 
Proposition 1 At the open-loop steady state equilibrium, party $i$ invests $k_{i}^{*}=\frac{\beta_{i}}{c(\rho+\delta)}$, and obtains $x_{i}^{*}=\frac{\beta_{i}-s \beta_{j}}{c(\rho+\delta) \delta}$. Party $i^{\prime}$ s steady state investment and consensus are both larger that party $j^{\prime}$ s if $\beta_{i}>\beta_{j}$.

Proof. In the open-loop formulation, the Hamiltonian for party (or candidate) $i$ rewrites as follows:

$$
\mathcal{H}_{i}(t)=e^{-\rho t}\left\{\beta_{i} x_{i}(t)-\frac{c}{2}\left[k_{i}(t)\right]^{2}+\lambda_{i}(t)\left[k_{i}(t)-s k_{j}(t)-\delta x_{i}\right]\right\}
$$

The first order conditions for the open-loop equilibrium are:

$$
\begin{aligned}
\frac{\partial \mathcal{H}_{i}(t)}{\partial k_{i}(t)} & =-c k_{i}(t)+\lambda_{i}(t)=0 \\
-\frac{\partial \mathcal{H}_{i}(t)}{\partial x_{i}(t)} & =\frac{\partial \lambda_{i}(t)}{\partial t}-\rho \lambda_{i}(t) \Rightarrow \\
\frac{\partial \lambda_{i}(t)}{\partial t} & =(\rho+\delta) \lambda_{i}(t)-\beta_{i}
\end{aligned}
$$

plus the initial conditions $x_{i}(0)=x_{i 0}$ and the transversality condition $\lambda_{i}(T)=$ 0 for all $i$.

From (7), one obtains $\lambda_{i}(t)=c k_{i}(t)$ and

$$
\frac{\partial k_{i}(t)}{\partial t} \propto \frac{\partial \lambda_{i}(t)}{\partial t}=c(\rho+\delta) k_{i}(t)-\beta_{i}
$$

The r.h.s. of the above expression is zero at

$$
k_{i}^{*}=\frac{\beta_{i}}{c(\rho+\delta)}
$$

which can be plugged into (1) to obtain

$$
\frac{\partial x_{i}(t)}{\partial t}=0 \text { at } x_{i}^{*}=\frac{\beta_{i}-s \beta_{j}}{c(\rho+\delta) \delta}
$$

It is then immediate to check that $\beta_{i}>\beta_{j}$ is both necessary and sufficient to ensure that $k_{i}^{*}>k_{j}^{*}$ and $x_{i}^{*}>x_{j}^{*}$.

Hence, a straightforward corollary to Proposition 1 is the following: 
Corollary 1 The candidate (or party) that attaches a larger weight to the ad interim utility of consensus invests more than the rival and ultimately wins the elections.

As to the stability analysis of the dynamic system, the following holds:

Proposition 2 The open-loop equilibrium $\left\{x_{i}^{*}, k_{i}^{*}\right\}$ is a saddle point.

Proof. See the Appendix.

Before passing on to the social planning case, a last remark is in order. Expression (9), describing the dynamics of party $i$ 's investment, reveals that it is independent of the rival's. This entails that, in model I, strategic interaction between parties takes place only through the dynamics of the state variable, as described by (1). This constrats with some of the acquired wisdom (see, e.g., Anderson and Prusa, 2001), where the efforts of parties are strategic complements. On this basis, whether parties overinvest or underinvest as compared to the social optimum is not obvious from the outset.

\subsection{The social optimum}

Now consider the setting where a benevolent social planner chooses the vector of investments $\left\{k_{i}(t)\right\}$ so as to maximise collective welfare, which is defined as the sum of both parties' discounted payoffs, under the constraint (1). This amounts to assuming that parties (or their candidates) are indeed representative of their electors, to the extent that those individuals who do not vote are irrelevant. ${ }^{7}$ In the present setting, the planner's behaviour is aimed at assessing the social convenience to put a ceiling to the parties' investments during the electoral campaign, even if this may well entail trading off some voters against a lower expenditure.

Now the relevant Hamiltonian is:

$$
\begin{aligned}
\mathcal{H}^{S P}(t)= & e^{-\rho t}\left\{\beta_{i} x_{i}(t)+\beta_{j} x_{j}(t)-\frac{c}{2}\left[k_{i}(t)\right]^{2}-\frac{c}{2}\left[k_{j}(t)\right]^{2}\right. \\
& \left.+\lambda_{i}(t)\left[k_{i}(t)-s k_{j}(t)-\delta x_{i}\right]+\lambda_{j}(t)\left[k_{j}(t)-s k_{i}(t)-\delta x_{j}\right]\right\}
\end{aligned}
$$

\footnotetext{
${ }^{7}$ This is somewhat similar to what is sometimes assumed in innovation race models, where the value of innovation is the same irrespectively of whether the firm racing for it is a private or a public one (see Kamien and Schwartz, 1982, and Reinganum, 1989, inter alia).
} 
where superscript $S P$ stands for social planning. The outcome is summarised by the following:

Proposition 3 At the social optimum, party $i$ invests $k_{i}^{S P}=\frac{\beta_{i}-s \beta_{i}}{c(\rho+\delta)}$, and obtains $x_{i}^{S P}=\frac{\beta_{i}\left(1+s^{2}\right)-2 s \beta_{j}}{c(\rho+\delta) \delta}$. Party $i^{\prime}$ s steady state investment and consensus are both larger that party $j^{\prime} s$ if $\beta_{i}>\beta_{j}$.

Proof. The first order conditions are:

$$
\begin{gathered}
\frac{\partial \mathcal{H}^{S P}(t)}{\partial k_{i}(t)}=-c k_{i}(t)+\lambda_{i}(t)-\lambda_{j}(t) s=0 \\
-\frac{\partial \mathcal{H}^{S P}(t)}{\partial x_{i}(t)}=\frac{\partial \lambda_{i}(t)}{\partial t}-\rho \lambda_{i}(t) \Rightarrow \\
\frac{\partial \lambda_{i}(t)}{\partial t}=(\rho+\delta) \lambda_{i}(t)-\beta_{i}
\end{gathered}
$$

plus of course the initial conditions $x_{i}(0)=x_{i 0}$ and the transversality condition $\lambda_{i}(T)=0$. From (13), we have:

$$
\lambda_{i}(t)=c k_{i}(t)+\lambda_{j}(t) s
$$

and

$$
\frac{d k_{i}(t)}{d t} \propto \frac{\partial \lambda_{i}(t)}{\partial t}-s \frac{\partial \lambda_{j}(t)}{\partial t}
$$

From (15) one obtains $\lambda_{i}(t)=c\left[k_{i}(t)+s k_{j}(t)\right] /\left(1-s^{2}\right)$. Using this and (14), (16) can be rewritten as follows:

$$
\frac{d k_{i}(t)}{d t} \propto c k_{i}(t)(\rho+\delta)-\beta_{i}+s \beta_{j}
$$

the r.h.s of (17) being zero at

$$
k_{i}^{S P}=\frac{\beta_{i}-s \beta_{i}}{c(\rho+\delta)}
$$

This equilibrium investment can be substituted into the dynamic equation of $x_{i}(t)$, to obtain

$$
\frac{\partial x_{i}(t)}{\partial t}=0 \text { at } x_{i}^{S P}=\frac{\beta_{i}\left(1+s^{2}\right)-2 s \beta_{j}}{c(\rho+\delta) \delta} .
$$


As in the case of Proposition 1, it is quickly checked that $\beta_{i}>\beta_{j}$ is both necessary and sufficient to ensure that $k_{i}^{S P}>k_{j}^{S P}$ and $x_{i}^{S P}>x_{j}^{S P}$.

Hence, under social planning the winner is the same candidate as in the social optimum. However, a thorough comparison between the two regimes remains to be carried out. In particular, one may wonder whether social planning may prevent parties from performing a wasteful effort duplication, and how this affects the total number of voters at equilibrium.

The stability properties of the steady state under social planning are stated in the following:

Proposition 4 The social planning equilibrium $\left\{x_{i}^{S P}, k_{i}^{S P}\right\}$ is a saddle point.

The proof is omitted, as it proceeds along the same lines as for Proposition 2 .

\subsection{Private vs social optimum}

Consider first equilibrium investments in advertising in the two regimes:

$$
k_{i}^{*}-k_{i}^{S P} \propto s \beta_{i}
$$

which entails that individual incentives leads to a socially wasteful duplication of efforts.

As to the equilibrium level of consensus, we have:

$$
X^{*}=x_{i}^{*}+x_{j}^{*}=\frac{\left(\beta_{i}+\beta_{j}\right)(1-s)}{c(\rho+\delta) \delta}
$$

and

$$
X^{S P}=x_{i}^{S P}+x_{j}^{S P}=\frac{\left(\beta_{i}+\beta_{j}\right)(1-s)^{2}}{c(\rho+\delta) \delta}
$$

with $X^{*}>X^{S P}$ for all admissible $s$.

The above discussion produces the following Corollary to Propositions 1-3:

Corollary 2 At the private optimum, candidates invest more than at the social optimum, for all admissible $\left\{s, \beta_{i}, \beta_{j}\right\}$. Accordingly, the number of voters is always higher at the private optimum than at the social optimum.

That is, the planner is happy with a lower number of voters, as long as this is more than compensated by a reduction in the wasteful duplication of efforts during the electoral campaign. 


\section{Model II}

When the stock of consensus evolves according to equation (4), it can be easily shown that the following holds:

Lemma 2 The open-loop solution is a degenerate closed-loop solution. Therefore, the open-loop equilibrium is subgame perfect.

Proof. See the Appendix.

Lemma 2 states that, as the open-loop equilibrium is strongly time consistent, it is subgame perfect. That is, as in model $\mathrm{I}$, the game is perfect or state redundant. In the remainder of the section, in order to concentrate on the comparison between the private and the social optima, I assume $\beta_{i}=\beta .^{8}$ This entails that the model can only produce symmetric equilibria and therefore cannot determine which party (or candidate) ultimately wins the elections.

The relevant open-loop Hamiltonian for party (or candidate) $i$ is:

$$
\mathcal{H}_{i}(t)=e^{-\rho t}\left\{\beta x_{i}(t)-c k_{i}(t)+\lambda_{i}(t)\left[\sqrt{k_{i}(t)-s k_{j}(t)}-\delta x_{i}(t)\right]\right\}
$$

where $\lambda_{i}(t)=\mu_{i}(t) e^{\rho t}$, and $\mu_{i}(t)$ is the co-state variable associated to $x_{i}(t)$.

\subsection{The private optimum}

The solution of the non-cooperative game between the two candidates leads to the following:

Proposition 5 At the open-loop steady state equilibrium, each party invests $k^{*}=\frac{\beta^{2}}{4 c^{2}(\rho+\delta)^{2}(1-s)}$, and obtains $x^{*}=\frac{\beta}{2 c(\rho+\delta) \delta}$.

Proof. The first order conditions for the open-loop equilibrium are:

$$
\frac{\partial \mathcal{H}_{i}(t)}{\partial k_{i}(t)}=-c+\frac{\lambda_{i}(t)}{2 \sqrt{k_{i}(t)-s k_{j}(t)}}=0
$$

\footnotetext{
${ }^{8}$ Although desirable in line of principle, keeping the asymmetry on $\beta_{i}^{\prime} s$ across parties yields an awkward expression for $\partial k_{i}(t) / \partial t$, which can be treated only numerically.
} 


$$
\begin{aligned}
-\frac{\partial \mathcal{H}_{i}(t)}{\partial x_{i}(t)} & =\frac{\partial \lambda_{i}(t)}{\partial t}-\rho \lambda_{i}(t) \Rightarrow \\
\frac{\partial \lambda_{i}(t)}{\partial t} & =(\rho+\delta) \lambda_{i}(t)-\beta
\end{aligned}
$$

plus the initial conditions $x_{i}(0)=x_{i 0}$ and the transversality condition $\lambda_{i}(T)=$ 0. From (24) one obtains:

$$
\lambda_{i}(t)=2 c \sqrt{k_{i}(t)-s k_{j}(t)}
$$

and

$$
k_{i}(t)=s k_{j}(t)+\frac{\left[\lambda_{i}(t)\right]^{2}}{4 c^{2}}
$$

which can be differentiated w.r.t. time to yield:

$$
\frac{d k_{i}(t)}{d t}=s \cdot \frac{\partial k_{j}(t)}{\partial t}+\frac{\lambda_{i}(t)}{2 c^{2}} \cdot \frac{\partial \lambda_{i}(t)}{\partial t}
$$

Imposing the symmetry conditions:

$$
k_{j}(t)=k_{i}(t) ; \frac{\partial k_{j}(t)}{\partial t}=\frac{\partial k_{i}(t)}{\partial t}
$$

and using (25) and (26), (28) rewrites as follows: ${ }^{9}$

$$
\frac{d k_{i}(t)}{d t} \propto 2 c(\rho+\delta) \sqrt{k_{i}(t)(1-s)}-\beta
$$

which is equal to zero at

$$
k^{*}=\frac{\beta^{2}}{4 c^{2}(\rho+\delta)^{2}(1-s)} .
$$

Then, substituting (31) into (4) and imposing that $\partial x_{i}(t) / \partial t=0$, one obtains:

$$
x^{*}=\frac{\beta}{2 c(\rho+\delta) \delta} .
$$

This proves the Proposition.

As to the dynamic properties of the steady state, the following can be shown to hold:

\footnotetext{
${ }^{9}$ The equation $d k_{i}(t) / d t=0$ has another root, $k_{i}(t)=0$, which can obviously be disregarded.
} 
Proposition 6 The open-loop equilibrium $\left\{x_{i}^{*}, k_{i}^{*}\right\}$ is a saddle point.

Proof. See the Appendix.

Moreover, on the basis of (27) and (28), it can be established that parties' investments are strategic complements, that is, any increase in $k_{i}(t)$ entails an increase (although less that proportional, due to the spillover effect) in $k_{j}(t)$, and conversely. Therefore, one can expect the private to be characterised by excess investment as compared to the socially optimal effort.

\subsection{The social optimum}

If a benevolent social planner chooses $k_{i}(t)$ and $k_{j}(t)$ to maximise social welfare, the relevant Hamiltonian is:

$$
\begin{aligned}
\mathcal{H}^{S P}(t)= & e^{-\rho t}\left\{\beta\left[x_{i}(t)+x_{j}(t)\right]-c\left[k_{i}(t)+k_{j}(t)\right]\right. \\
& \left.+\lambda_{i}(t)\left[\sqrt{k_{i}(t)-s k_{j}(t)}-\delta x_{i}\right]+\lambda_{j}(t)\left[\sqrt{k_{j}(t)-s k_{i}(t)}-\delta x_{j}\right]\right\}
\end{aligned}
$$

The outcome is summarised by the following:

Proposition 7 At the social optimum, each party invests $k^{S P}=\frac{\beta^{2}(1-s)}{4 c^{2}(\rho+\delta)^{2}}$, and obtains $x^{S P}=\frac{\beta(1-s)}{2 c(\rho+\delta) \delta}$.

Proof. The first order conditions are:

$$
\begin{gathered}
\frac{\partial \mathcal{H}^{S P}(t)}{\partial k_{i}(t)}=-c+\frac{\lambda_{i}(t)}{2 \sqrt{k_{i}(t)-s k_{j}(t)}}+\frac{s \lambda_{j}(t)}{2 \sqrt{k_{j}(t)-s k_{i}(t)}} \\
-\frac{\partial \mathcal{H}^{S P}(t)}{\partial x_{i}(t)}=\frac{\partial \lambda_{i}(t)}{\partial t}-\rho \lambda_{i}(t) \Rightarrow \\
\frac{\partial \lambda_{i}(t)}{\partial t}=(\rho+\delta) \lambda_{i}(t)-\beta
\end{gathered}
$$

Now, imposing symmetry, I obtain:

$$
\begin{aligned}
& \lambda(t)=\frac{2 c \sqrt{k(t)(1-s)}}{1-s} \\
& k(t)=\frac{[\lambda(t)]^{2}(1-s)}{4 c^{2}}
\end{aligned}
$$


from which:

$$
\frac{\partial k(t)}{\partial t} \propto \frac{\partial \lambda(t)}{\partial t}=\frac{2 c(\rho+\delta) \sqrt{k(t)(1-s)}}{1-s}-\beta
$$

Therefore, ${ }^{10}$

$$
\frac{\partial k(t)}{\partial t}=0 \text { at } k^{S P}=\frac{\beta^{2}(1-s)}{4 c^{2}(\rho+\delta)^{2}}
$$

and

$$
\frac{\partial x(t)}{\partial t}=\frac{\beta(1-s)}{2 c(\rho+\delta)}-\delta x(t)=0 \text { at } x^{S P}=\frac{\beta(1-s)}{2 c(\rho+\delta) \delta} .
$$

This concludes the proof.

The stability properties of the system at the steady state produced by social planning are as follows:

Proposition 8 The social planning equilibrium $\left\{x^{S P}, k^{S P}\right\}$ is a saddle point.

The proof is omitted for brevity, as it is analogous to the proof of Proposition 6 .

\subsection{Private vs social optimum}

The comparative evaluation of the two regimes can be quicly dealt with, as it is qualitatively analogous to section 3.3. Evaluating Propositions 5 and 7, it is immediate to verify that

$$
k^{S P}<k^{*} ; x^{S P}<x^{*} .
$$

Accordingly, as in model I, also here the social optimum is achieved by invesing less that in the private optimum. As a consequance, the socially efficient number of voters is lower under social planning than at the private equilibrium.

\footnotetext{
${ }^{10}$ Again, the solution $k(t)=0$ can be dismissed.
} 


\section{Extension of model I: optimal fragmenta- tion}

In the foregoing analysis, I have adopted the assumption that there exist only two parties, and I have evaluated the efficiency of such a system. However, multiparty systems are rather common, and to this regard several interesting questions can be addressed in a generalisation of any of the above settings. One such question is whether there should be a limit to the number of parties, and, if so, how to set this limit. Two related questions are (i) whether the optimal number of parties obtains by maximising the overall number of voters or collective welfare, and (ii) whether optimal fragmentation is higher in the social or in the private optimum. In particular, question (ii) involves the comparison of two situations. One is the first best where a benevolent social planner controls both the number of parties and their individual investment in the electoral campaign. The other is a second best where the investment is noncooperatively decided by parties, while the number of parties is controlled by a benevolent planner.

Here I reconsider model I with $n$ parties (and $n$ candidates), where, for the sake of simplicity, I adopt the symmetry assumption $\beta_{i}=\beta$ for all $i$. This amounts to excluding the use of a quorum so as to determine the optimal number of parties (or candidates), since the equilibrium size (i.e., the volume of votes in steady state) is the same across parties.

The differential equation of the state variable is:

$$
\frac{\partial x_{i}(t)}{\partial t}=k_{i}(t)-s \sum_{j \neq i} k_{j}(t)-\delta x_{i}
$$

Accordingly, the closed-loop formulation of candidate $i^{\prime}$ s Hamiltonian now rewrites as:

$$
\begin{aligned}
\mathcal{H}_{i}(t)= & e^{-\rho t}\left\{\beta x_{i}(t)-\frac{c}{2}\left[k_{i}(t)\right]^{2}+\lambda_{i i}(t)\left[k_{i}(t)-s \sum_{j \neq i} k_{j}(t)-\delta x_{i}\right]\right. \\
& \left.+\sum_{j \neq i} \lambda_{i j}(t)\left[k_{j}(t)-s \sum_{m \neq j} k_{m}(t)-\delta x_{j}\right]\right\}
\end{aligned}
$$

Once again, it can be shown that the open-loop solution is a degenerate closed-loop solution, and therefore (42) can be reformulated by setting $\lambda_{i i}(t)=$ $\lambda_{i}(t)$ and $\lambda_{i j}(t)=0$ for all $j$. 
Proposition 9 In the private optimum with $n$ candidates, the overall number of voters is maximised at $n_{x}^{*}=\frac{1+s}{2 s}$, while the equilibrium collective utility is maximised at $n_{\Pi}^{*}=\frac{\delta(1+2 s)+2 \rho(1+s)}{4 s(\rho+\delta)}$, with $n_{x}^{*}>n_{\Pi}^{*}$ in the admissible range of parameters.

Proof. First order conditions for the private optimum are:

$$
\begin{aligned}
\frac{\partial \mathcal{H}_{i}(t)}{\partial k_{i}(t)} & =-c k_{i}(t)+\lambda_{i}(t)=0 \\
-\frac{\partial \mathcal{H}_{i}(t)}{\partial x_{i}(t)} & =\frac{\partial \lambda_{i}(t)}{\partial t}-\rho \lambda_{i}(t) \Rightarrow \\
\frac{\partial \lambda_{i}(t)}{\partial t} & =(\rho+\delta) \lambda_{i}(t)-\beta
\end{aligned}
$$

plus the same initial and transversality conditions as in section 3.1. From (41), (43) and (44) I obtain: ${ }^{11}$

$$
k_{i}^{*}=\frac{\beta}{c(\rho+\delta)} ; x_{i}^{*}=\frac{\beta[1-s(n-1)]}{c(\rho+\delta) \delta}>0 \forall n<\bar{n}=\frac{1+s}{s}
$$

Hence, the total number of voters at equilibrium is $X^{*}=n x_{i}^{*}$, while the collective payoff accruing to parties is

$$
\Pi^{*}=n\left[\beta x_{i}^{*}-\frac{c}{2}\left(k_{i}^{*}\right)^{2}\right]=\frac{\beta^{2} n[\delta(1+2 s(1-n))+2 \rho(1+s(1-n))]}{2 c(\rho+\delta)^{2} \delta}
$$

It is then immediate to verify that $X^{*}$ is maximised at

$$
n_{x}^{*}=\frac{1+s}{2 s}<\bar{n}
$$

while $\Pi^{*}$ is maximised at

$$
n_{\Pi}^{*}=\frac{\delta(1+2 s)+2 \rho(1+s)}{4 s(\rho+\delta)}<\bar{n}
$$

\footnotetext{
${ }^{11}$ I omit computational details for brevity. It can be also easily shown that (45) is a saddle point.
} 
with

$$
n_{x}^{*}-n_{\Pi}^{*}=\frac{\delta}{4 s(\rho+\delta)}>0 \text { for all } s, \delta, \rho .
$$

This concludes the proof.

The above result tells that, at the privately optimal steady state, the fragmentation that ensures the highest collective utility produces too little participation to elections on the part of voters. This, in turn, calls for the evaluation of the same issue under social planning.

Proposition 10 Under social planning with $n$ candidates, both the overall number of voters and the collective utility are maximised at $n^{S P}=\frac{1+s}{3 s}$.

Proof. The benevolent social planner maximises

$$
\mathcal{H}^{S P}(t)=e^{-\rho t}\left\{\beta x(t)-\frac{c}{2}[k(t)]^{2}+\lambda(t)[k(t)(1-s(n-1))-\delta x]\right\}
$$

w.r.t. individual advertising effort $k(t)$. The usual procedure yields the following saddle point equilibrium:

$$
k^{S P}=\frac{\beta[1-s(n-1)]}{c(\rho+\delta)}>0 \forall n<\bar{n}=\frac{1+s}{s} ; x^{S P}=\frac{\beta[1-s(n-1)]^{2}}{c(\rho+\delta) \delta}
$$

These values allows me to calculate

$$
X^{S P}=\frac{n \beta[1-s(n-1)]^{2}}{c(\rho+\delta) \delta}
$$

which is maximised at

$$
n_{x}^{S P}=\frac{1+s}{3 s}<\bar{n}
$$

Finally, routine calculations are needed to verify that $n_{x}^{S P}$ also maximises

$$
\Pi^{S P}=n\left[\beta x^{S P}-\frac{c}{2}\left(k^{S P}\right)^{2}\right]
$$

and therefore $n_{\Pi}^{S P}=n_{x}^{S P}=n^{S P}$.

A relevant consequence of Propositions 9-10 is:

Corollary 3 For all $s \in\left[0, \frac{1}{2}\right)$, 
- $n_{x}^{*}>n^{S P}>n_{\Pi}^{*}$ iff $\frac{\delta}{\rho}>\frac{2(1+s)}{1-2 s}$

- $n_{x}^{*}>n_{\Pi}^{*}>n^{S P}$ iff $\frac{\delta}{\rho} \in\left[0, \frac{2(1+s)}{1-2 s}\right)$

For all $s \in\left(\frac{1}{2}, 1\right], n_{x}^{*}>n^{S P}>n_{\Pi}^{*}$ for all $\delta, \rho$.

Proof. From Proposition 9 we already know that $n_{x}^{*}>n_{\Pi}^{*}$. Moreover, $n_{x}^{*}>n^{S P}$ always. To complete the proof, observe that

$$
n^{S P}-n_{\Pi}^{*}=\frac{\delta(1-2 s)-2 \rho(1+s)}{12 s(\rho+\delta)}>0 \text { iff } \frac{\delta}{\rho}>\frac{2(1+s)}{1-2 s}
$$

where

$$
\frac{2(1+s)}{1-2 s} \geq 2 \text { and increasing in } s, \text { for all } s \in\left[0, \frac{1}{2}\right)
$$

while it is negative for all $s \in\left(\frac{1}{2}, 1\right]$. Therefore, in the latter interval, $n^{S P}-n_{\Pi}^{*}>0$ for all $\delta, \rho$.

Now examine the two alternative scenarios. The first is social planning, where a benevolent dictator controls the advertising investment of each party, and maximises collective welfare in steady state w.r.t. $n$. The second scenario is a private equilibrium driven by individual incentives, with the planner controlling only the number of parties. Needless to say, the welfare level associated to the first equilibrium is strictly larger than that associated with the second. However, the socially optimal fragmentation depends upon the size of parameters. In particular, it is higher under planning than in the private optimum (i) for all values of parameters describing the intertemporal properties of the model, if the negative externality is large; and (ii) if the depreciation rate $\delta$ is considerably larger than the discount rate $\rho$, when the negative externality is low.

Result (i) indicates that having a large number of candidates (or parties) is socially preferable when each party's advertising expenditure is highly effective in stealing voters from the rivals, while result (ii) states that a large number of candidates is socially efficient when the opposite holds, provided that the perceived duration of the game (as measured by $\rho$ ) is low enough compared to the decay rate of the stock of each party's consensus. 


\section{Extension of model II: optimal fragmenta- tion}

An analogous exercise can be carried out under the dynamics (4), by assuming that $n$ symmetric candidates race for the office. As the procedure closely replicates what I have just exposed in section 5 , here I will confine myself to the main elements.

The open-loop Hamiltonian of party $i$ is:

$$
\mathcal{H}_{i}(t)=e^{-\rho t}\left\{\beta x_{i}(t)-c k_{i}(t)+\lambda_{i}(t)\left[\sqrt{k_{i}(t)-s \sum_{j \neq i} k_{j}(t)}-\delta x_{i}(t)\right]\right\}
$$

while the Hamiltonian of the social planner is simply $\mathcal{H}^{S P}(t)=\sum_{i=1}^{n} \mathcal{H}_{i}(t)$. As the open-loop equilibrium is subgame perfect, the closed-loop formulation of the problem is redundant. On these bases, the following results can be shown to hold. ${ }^{12}$

Proposition 11 In the private optimum with $n$ candidates, the overall number of voters is $X^{*}=\frac{n \beta}{2 c(\rho+\delta) \delta}$, which is everywhere uncreasing in $n$. The equilibrium collective utility is

$$
\Pi^{*}=\frac{n \beta^{2}}{2 c(\rho+\delta)}\left\{\frac{1}{\delta}-\frac{1}{2(\rho+\delta)[1-s(n-1)]}\right\}
$$

which takes its global maximum at $n_{\Pi}^{*}=\frac{[2(\rho+\delta)-\delta](1+s)}{2 s(\rho+\delta)}$.

Proposition 12 Under social planning with $n$ candidates, the overall number of voters in steady state is

$$
X^{S P}=\frac{\beta n[1-s(n-1)]}{2 c(\rho+\delta) \delta}
$$

and the associated collective payoff is

$$
\Pi^{S P}=\frac{n \beta^{2}[1-s(n-1)](2 \rho+\delta)}{4 c(\rho+\delta)^{2} \delta} .
$$

\footnotetext{
${ }^{12}$ The proofs are omitted for the sake of brevity. They are available from the author upon request.
} 
Both the overall number of voters and the collective utility are maximised at $n^{S P}=\frac{1+s}{2 s}$.

Propositions 11-12 produce the following:

Corollary $4 n_{\Pi}^{*}>n^{S P}$ for all $\{\delta, \rho\}$ and all $s<1 /(n-1)$.

That is, in model II the second best optimal fragmentation is surely higher that the socially optimal fragmentation. Corollaries 3 and 4 indicate that the optimal number of parties (or candidates ) is sensitive to the shape of the advertising technology used for the electoral campaign. If decreasing returns appear in the instantaneous cost (as in model I), than anything may happen, depending upon the relative size of parameters. If instead decreasing returns affect the dynamics of consensus (as in model II), then it clearly emerges that the maximisation of collective welfare at the private optimum involves too many parties as compared to the socially efficient situation.

\section{Concluding remarks}

I have analysed two differential games describing electoral campaigns where two candidates invest so as to increase the number of their respective voters. The outcomes of the non-cooperative games have been evaluated against the social optimum, where a benevolent social planner chooses the investment levels so as to maximise collective welfare. In both games, the private optimum is characterised by overinvestment and a larger number of voters as compared to the social optimum. Therefore, it appears that, beyond some point, the social benefit associated with having more electors expressing their opinion at the polls is not worth the cost that must be borne for convincing them to vote.

Then, I have extended both models to account for $n$ candidates, in order to evaluate what is the optimal number of candidates (or parties), on a welfare basis. This analysis has produced non-univocal results, in that the first best number of candidates (i.e., the number that maximises social welfare when the planner also controls directly the parties' efforts) may be higher or lower than the second best number of candidates (i.e., the number that maximises social welfare given the non-cooperative investment behaviour of parties), depending on the shape of cost functions and the dynamics of consensus. If the instantaneous cost of investing is convex and the dynamics of 
accumulation is linear, then the number of parties at the first best is higher (lower) than the number of parties at the second best, if the spillover effect is large (low) enough. If instead the cost of investment is linear, the first best number of parties is always lower than the second best number of parties, irrespectively of the spillover level. 


\section{Appendix}

Proof of Lemma 1. The first order conditions for the closed-loop equilibrium are:

$$
\begin{gathered}
\frac{\partial \mathcal{H}_{i}(t)}{\partial k_{i}(t)}=-c k_{i}(t)+\lambda_{i i}(t)-\lambda_{i j}(t) s=0 \\
-\frac{\partial \mathcal{H}_{i}(t)}{\partial x_{i}(t)}-\frac{\partial \mathcal{H}_{i}(t)}{\partial k_{j}(t)} \cdot \frac{\partial k_{j}^{*}(t)}{\partial x_{i}(t)}=\frac{\partial \lambda_{i i}(t)}{\partial t}-\rho \lambda_{i i}(t) \forall i, j
\end{gathered}
$$

plus the initial conditions $x_{i}(0)=x_{i 0}$ and the transversality condition $\lambda_{i i}(T)=$ 0 , which entails $\lambda_{i i}(T) S\left[x_{i}(T)\right]=0$.

To prove the Lemma, it suffices to observe that (a1) does not contain the state variables. Therefore, its solution with respect to $k_{i}(t)$ yields $k_{i}^{*}(t)=$ $\left[\lambda_{i i}(t)-\lambda_{i j}(t) s\right] / c$, from which we immediately obtain

$$
\frac{\partial k_{i}^{*}(t)}{\partial x_{j}(t)}=0
$$

This shows that (i) the solution $\lambda_{i j}(t)=0$ is admissible; accordingly (ii) the open-loop solution is a degenerate closed-loop solution.

Proof of Proposition 2. The relevant dynamic equations are:

$$
\begin{gathered}
\frac{\partial x_{i}(t)}{\partial t} \equiv \dot{x}_{i}=k_{i}(t)-s k_{j}(t)-\delta x_{i}(t) \\
\frac{\partial k_{i}(t)}{\partial t} \equiv \dot{k}_{i}=\frac{1}{c}\left[c(\rho+\delta) k_{i}(t)-\beta_{i}\right]
\end{gathered}
$$

and the stability properties of the system (a4-a5) depend upon the sign of the trace and determinant of the following Jacobian matrix:

$$
\Xi=\left[\left.\begin{array}{cc}
\frac{\partial \dot{x}_{i}}{\partial x_{i}}=-\delta & \frac{\partial \dot{x}_{i}}{\partial k_{i}}=1 \\
\frac{\partial \dot{k}_{i}}{\partial x_{i}}=0 & \frac{\partial \dot{k}_{i}}{\partial k_{i}}=\rho+\delta
\end{array}\right|_{x_{i}^{*}, k_{i}^{*}}\right.
$$

with $\operatorname{Tr}(\Xi)=\rho>0$ and $\Delta(\Xi)=-\delta(\rho+\delta)<0$. Since the determinant is everywhere positive, $\left\{x_{i}^{*}, k_{i}^{*}\right\}$ is a saddle point. 
Proof of Lemma 2. The closed-loop Hamiltonian of party $i$ is:

$$
\begin{aligned}
\mathcal{H}_{i}(t)= & e^{-\rho t}\left\{\beta_{i} x_{i}(t)-\frac{c}{2}\left[k_{i}(t)\right]^{2}+\lambda_{i i}(t)\left[\sqrt{k_{i}(t)-s k_{j}(t)}-\delta x_{i}(t)\right]\right. \\
& \left.+\lambda_{i j}(t)\left[\sqrt{k_{j}(t)-s k_{i}(t)}-\delta x_{i}(t)\right]\right\}
\end{aligned}
$$

and the first order condition on $k_{i}(t)$ is:

$$
\frac{\partial \mathcal{H}_{i}(t)}{\partial k_{i}(t)}=-c+\frac{\lambda_{i i}(t)}{2 \sqrt{k_{i}(t)-s k_{j}(t)}}-\frac{s \lambda_{i j}(t)}{2 \sqrt{k_{j}(t)-s k_{i}(t)}}
$$

Now, from (a7) it is immediate to establish that

$$
\frac{\partial^{2} \mathcal{H}_{i}(t)}{\partial k_{i}(t) \partial x_{j}(t)}=0 \forall i, j
$$

which proves the Lemma.

Proof of Proposition 6. Having imposed symmetry, the relevant dynamic equations are:

$$
\begin{gathered}
\frac{\partial x(t)}{\partial t} \equiv \dot{x}=\sqrt{k(t)(1-s)}-\delta x(t) \\
\frac{\partial k(t)}{\partial t} \equiv \dot{k}=\frac{[4 c(\delta+\rho) \sqrt{k(t)(1-s)}-\beta] \sqrt{k(t)(1-s)}}{c(1-s)}
\end{gathered}
$$

and the stability properties of the system (a9-a10) depend upon the sign of the trace and determinant of the following Jacobian matrix:

$$
\Xi=\left.\left[\begin{array}{cc}
\frac{\partial \dot{x}}{\partial x}=-\delta & \frac{\partial \dot{x}}{\partial k}=\frac{1-s}{2 \sqrt{k(t)(1-s)}} \\
\frac{\partial \dot{k}}{\partial x}=0 & \frac{\partial \dot{k}_{i}}{\partial k_{i}}=2(\rho+\delta)-\frac{\beta \sqrt{k(t)(1-s)}}{2 c(1-s) k(t)}
\end{array}\right]\right|_{x^{*}, k^{*}}
$$

with

$$
\begin{gathered}
\operatorname{Tr}(\Xi)=\delta+2 \rho-\frac{\beta \sqrt{k(t)(1-s)}}{2 c(1-s) k(t)} \\
\Delta(\Xi)=-\delta\left[2(\rho+\delta)-\frac{\beta \sqrt{k(t)(1-s)}}{2 c(1-s) k(t)}\right]
\end{gathered}
$$

which, evaluated at $\left\{x^{*}, k^{*}\right\}$, yield $\operatorname{Tr}(\Xi)=\rho>0$ and $\Delta(\Xi)=-\delta(\rho+\delta)<$ 0 . Since the determinant is everywhere positive, $\left\{x^{*}, k^{*}\right\}$ is a saddle point. 


\section{References}

[1] Abramovitz, A.I. (1991), "Incumbency, Campaign Spending, and the Decline of Competition in the US House Elections", Journal of Politics, 53, 34-56.

[2] Adams, J. (1999), "Policy Divergence in Multicandidate Probabilistic Spatial Voting", Public Choice, 100, 103-22.

[3] Adams, J. (2000), "Multicandidate Equilibrium in American Elections", Public Choice, 103, 297-325.

[4] Anderson, J.E. and T.J. Prusa (2001), "Political Market Structure", NBER working paper no. 8371.

[5] Anderson, S.P., A. Kats and J.-F. Thisse (1994), "Probabilistic Voting and Platform Selection in Multi-party Elections", Social Choice and Welfare, 11, 305-22.

[6] Ansolabehere, S. and J.M. Snyder, Jr. (2000), "Valence Politics and Equilibrium in Spatial Election Models", Public Choice, 103, 327-36.

[7] Austen-Smith, D. (1995), "Campaign Contributions and Access", American Political Science Review, 89, 566-81.

[8] Baron, D. (1989), "Service-induced Campaign Contributions and the Electoral Equilibrium", Quarterly Journal of Economics, 104, 45-72.

[9] Bartholdi J.J., L.S. Narasimhan and C.A. Tovey (1991), "Recognizing Majority-Rule Equilibrium in Spatial Voting Games", Social Choice and Welfare, 8, 183-97.

[10] Başar, T. and G.J. Olsder (1982, 1995²), Dynamic Noncooperative Game Theory, San Diego, Academic Press.

[11] Brander, J. and B. Spencer (1983), "Strategic Commitment with R\&D: The Symmetric Case", Bell Journal of Economics, 14, 225-35.

[12] Cellini, R. and L. Lambertini (2001), "A Differential Game Approach to Investment in Product Differentiation", Journal of Economic Dynamics and Control, forthcoming. 
[13] Clemhout, S. and H.Y. Wan, Jr. (1974), "A Class of Trilinear Differential Games", Journal of Optimization Theory and Applications, 14, 419-24.

[14] Dixon, H.D. (1985), "Strategic Investment in an Industry with a Competitive Product Market", Journal of Industrial Economics, 33, 483-99.

[15] Dockner, E.J., G. Feichtinger and S. Jørgensen (1985), "Tractable Classes of Nonzero-Sum Open-Loop Nash Differential Games: Theory and Examples", Journal of Optimization Theory and Applications, 45, 179-97.

[16] Dockner, E.J, S. Jørgensen, N. Van Long and G. Sorger (2000), Differential Games in Economics and Management Science, Cambridge, Cambridge University Press.

[17] Feichtinger, G. (1983), "The Nash Solution of an Advertising Differential Game: Generalization of a Model by Leitmann and Schmitendorf", IEEE Transactions on Automatic Control, 28, 1044-48.

[18] Feichtinger, G., R.F. Hartl and P.S. Sethi (1994), "Dynamic Optimal Control Models in Advertising: Recent Developments", Management Science, 40, 195-226.

[19] Fershtman, C. (1984), "Goodwill and Market Shares in Oligopoly", Economica, 51, 271-82.

[20] Fershtman, C. (1987), "Identification of Classes of Differential Games for Which the Open-Loop is a Degenerated Feedback Nash Equilibrium", Journal of Optimization Theory and Applications, 55, 217-31.

[21] Fershtman, C. and S. Nitzan (1991), "Dynamic Voluntary Provision of Public Goods", European Economic Review, 35, 1057-67.

[22] Fershtman, C., M.I. Kamien and E. Muller (1992), "Integral Games: Theory and Applications", in Feichtinger, G. (ed.), Dynamic Economic Models and Optimal Control, Amsterdam, North-Holland, 297-311.

[23] Friedman, J.W. (1983), "Advertising and Oligopolistic Equilibrium", Bell Journal of Economics, 14, 464-73.

[24] Harris, C. and J. Vickers (1985), "Perfect Equilibrium in a Model of a Race", Review of Economic Studies, 52, 193-209. 
[25] Harris, C. and J. Vickers (1987), "Racing with Uncertainty", Review of Economic Studies, 54, 1-21.

[26] Kamien, K.I. and N.L. Schwartz (1982), Market Structure and Innovation, Cambridge, Cambridge University Press.

[27] Jacobsen, G.C. (1978), "The Effects of Campaign Spending in Congressional Elections", American Political Science Review, 72, 469-91.

[28] Jacobsen, G.C. (1980), Money in Congressional Elections, New Haven, Yale University Press.

[29] Jacobsen, G.C. (1987), "The Marginals Never Vanished: Incumbency and Competition in the Elections to the US House of Representatives, 1952-1982", American Journal of Political Science, 31, 126-41.

[30] Jørgensen, S. (1982), "A Survey of Some Differential Games in Advertising", Journal of Economic Dynamics and Control, 4, 341-69.

[31] Leitmann, G. and W.E. Schmitendorf (1978), "Profit Maximization through Advertising: A Nonzero Sum Differential Game Approach", IEEE Transactions on Automatic Control, 23, 646-50.

[32] Martin, S. (1993), Advanced Industrial Economics, Oxford, Blackwell.

[33] Mehlmann, A. (1988), Applied Differential Games, New York, Plenum Press.

[34] Mehlmann, A. and R. Willing (1983), "On Nonunique Closed-Loop Nash Equilibria for a Class of Differential Games with a Unique and Degenerate Feedback Solution", Journal of Optimization Theory and Applications, 41, 463-72.

[35] Page, S.E., K. Kollman and J.H. Miller (1993), "Adaptive Parties and Spatial Voting Theory", in Grofman, B. (ed.), Information, Participation, and Choice: An Economic Theory of Democracy in Perspective, Ann Arbor, University of Michigan Press, 161-72.

[36] Persson, T. and G. Tabellini (2000), Political Economics: Explaining Economic Policy, Zeuthen Lecture Book Series, Cambridge, MA, MIT Press. 
[37] Reinganum, J. (1981) "Dynamic Games of Innovation", Journal of Economic Theory, 25, 21-41.

[38] Reinganum, J. (1982a), "A Dynamic Game of R\&D: Patent Protection and Competitive Behavior", Econometrica, 50, 671-88.

[39] Reinganum, J. (1982b), "A Class of Differential Games for Which the Closed Loop and Open Loop Nash Equilibria Coincide", Journal of Optimization Theory and Applications, 36, 253-62.

[40] Reinganum, J. (1989), "The Timing of Innovation: Research, Development and Diffusion", in R. Schmalensee and R. Willig (eds.), Handbook of Industrial Organization, vol. 1, Amsterdam, North-Holland.

[41] Tirole, J. (1988), The Theory of Industrial Organization, Cambridge, MA, MIT Press.

[42] Weber, S. (1998), "Entry Deterrence in Electoral Spatial Competition", Social Choice and Welfare, 15, 31-56. 